\title{
Análise do acesso aos serviços odontológicos públicos no Brasil
}

\author{
Analysis of public dental services access in Brazil \\ Emílio Prado da Fonseca ${ }^{1,2}$, Suelen Garcia Oliveira da Fonseca², Marcelo de Castro Meneghim³ \\ 'Secretaria Municipal de Saúde, Prefeitura de Divinópolis (PMD) - Divinópolis (MG), Brasil. \\ ${ }^{2}$ Programa de Pós-Graduação em Odontologia, Faculdade de Odontologia de Piracicaba (FOP/UNICAMP) - Piracicaba(SP), Brasil. \\ ${ }^{3}$ Departamento de Odontologia Social e Preventiva, FOP/UNICAMP - Piracicaba (SP), Brasil.
}

DOI: http://dx.doi.org/10.7322/abcshs.v42i2.1008

\section{RESUMO}

O acesso à saúde bucal tem sido analisado como parte do processo de integralidade do cuidado. Este trabalho teve como objetivo revisar a literatura científica brasileira sobre acesso à saúde bucal entre 2007 e 2014. A partir da pergunta formulada, foram revisadas as bases de dados PubMed/MEDLINE, LILACS, SciELO, Scopus e BIREME/BVS. Além de aspas para palavras compostas, para serem recuperadas juntas, foram utilizados operadores booleanos para ampliar ou especificar a pesquisa em busca da melhor informação. Foram selecionados descritores válidos e aplicados os filtros: texto completo, idiomas (inglês e português), ano, assunto e tipo de documento (artigo completo). Fizeram parte da análise 35 artigos. Houve uma grande diversidade no percurso metodológico (desenho) dos estudos, sendo 24 (68,57\%) do tipo epidemiológico transversal e $5(14,28 \%)$ do tipo epidemiológico ecológico. O maior nível de evidência científica encontrado foi do tipo ensaio comunitário, utilizado em apenas 1 (2,85\%) artigo. Não foi encontrado nenhum artigo do tipo epidemiológico longitudinal. No presente estudo, identificou-se o acesso à saúde bucal como um dispositivo transformador da realidade, modulado por aspectos contextuais, de serviço e individuais.

Palavras-chave: saúde bucal; acesso aos serviços de saúde; Sistema Único de Saúde.

\section{ABSTRACT}

Access to oral health has been examined as part of the process of integrality in health care. This paper aimed to review the Brazilian scientific literature on access to oral health between 2007 and 2014. From the elaborated question, these databases were reviewed: PubMed/MEDLINE, LILACS, SciELO, Scopus and Bireme/BVS. In addition to the use of quotation marks for compound words to be retrieved together,boolean operators were applied to expand or specify the search for the best information. Valid descriptors were selected and, then, filters applied: full text, languages (English and Portuguese), year, subject and type of document (full article). A total of 35 articles were selected. There was a great diversity in methodology of the studies, as 24 (68.57\%) were cross section and 5 (14.28\%) were articles with an ecological approach. The highest level of scientific evidence found was the community trial and was used in only one article (2.85\%). It was not found any longitudinal article. It was possible to identify the access to oral health as a device transformer of reality, modulated by contextual, services and individuals factors.

Keywords: oral health; health services accessibility; Unified Health System. 


\section{INTRODUÇÃO}

Ao longo dos últimos 20 anos, o acesso à saúde bucal tem sido relacionado com o uso, a utilização, a acessibilidade e a porta de entrada em um serviço assistencial de saúde ${ }^{1,2}$. No contexto brasileiro, a universalização do acesso constitui um dos principais desafios da gestão dos sistemas locais de saúde enfrentados para a efetivação do Sistema Único de Saúde (SUS) como modelo de política pública ${ }^{3}$. Em sentido mais amplo, o acesso à saúde bucal abrange fatores políticos, organizacionais, contextuais e simbólicos, e é objeto de discussão como parte do processo de geração da integralidade do cuidado à saúde $\mathrm{e}^{3,4}$.

Observa-se na literatura sobre o tema "acesso" uma variação em relação ao momento de entrada no serviço para os resultados dos cuidados recebidos, demandados e em relação ao nível de atenção ${ }^{1-4}$. Nesse contexto, questões vinculadas a porte populacional, políticas de saúde bucal, estrutura do serviço, contexto social, fatores econômicos, aspectos demográficos e comportamentos coletivo e individual têm forte influência sobre o modelo político, organizacional e de assistência à saúde bucal ${ }^{1-5}$. A compreensão dos fatores que constrangem e potencializam o acesso à saúde bucal permite $\mathrm{o}$ monitoramento e o gerenciamento do sistema de saúde para impulsionar mudanças comportamentais e melhores resultados das ações oferecidas pelos serviços e sistemas de saúde. Sendo assim, é necessário o refinamento do conhecimento dos mecanismos de como esses fatores interagem e atuam sobre a saúde bucal de indivíduos e populações. É importante também o acesso como elemento transformador da realidade por meio da consciência de cidadania e do sentimento de pertencimento e da contribuição para a organização dos serviços locais de saúde bucal ${ }^{2,3,5}$.

Dessa forma, o objetivo deste estudo foi revisar a literatura sobre acesso à saúde bucal entre 2007 e 2014.

\section{MÉTODOS}

Trata-se de um estudo do tipo revisão de literatura pelo método integrado. A primeira etapa do estudo foi a formulação de uma pergunta para subsidiar a busca eletrônica em bases de dados. Assim, a pergunta formulada foi: "As condições políticas, socioeconômicas, de gestão do serviço e do indivíduo interferem no acesso à saúde bucal?"

A partir da pergunta formulada, foram revisadas as bases de dados PubMed, da Medical Literature Analysis and Retrieval System Online (MEDLINE); Literatura Latino-Americana e do Caribe em Ciências da Saúde (LILACS); Scientific Electronic Library Online
(SciELO); Scopus e Biblioteca Virtual em Saúde (BVS), do Centro Latino-Americano e do Caribe de Informação em Ciências da Saúde (BIREME). Foram utilizados os operadores booleanos "OR" e "AND", que permitem ampliar ou especificar a pesquisa em busca da melhor informação sobre determinado assunto. Além disso, foram colocadas aspas para palavras compostas, a fim de serem recuperadas juntas. Foram selecionados os seguintes descritores válidos: "acesso"; "acesso aos serviços de saúde"; "acesso universal a serviços de saúde"; "utilização"; "saúde bucal"; "desigualdades em saúde"; "políticas públicas de saúde"; "serviços de saúde bucal"; "avaliação do desempenho de sistemas de saúde"; "avaliação dos serviços de saúde"; "avaliação em saúde". Também foram aplicados os filtros: texto completo, idiomas (inglês e português), ano, assunto e tipo de documento (artigo completo).

$\mathrm{Na}$ primeira etapa da revisão de literatura, foram lidos os títulos e os resumos dos artigos encontrados nas buscas realizadas nas bases de dados entre 2007 e 2014. Adotou-se como critério inicial de seleção dos artigos o ano de 2007, para poder captar os impactos do aumento de oferta de serviços públicos odontológicos especializados e não especializados, que ocorreu por meio da reestruturação da Política Nacional de Saúde Bucal (PNSB) ${ }^{6,7}$. Em seguida, os artigos remanescentes da primeira fase foram lidos na íntegra e os artigos duplicados ou que não se enquadraram no escopo do tema acesso à saúde bucal foram excluídos. Um pesquisador doutor em saúde coletiva foi consultado em caso de dúvida sobre a inclusão ou exclusão de um artigo. Também foram consultados documentos como a Pesquisa Nacional de Amostras por Domicílio (PNAD), o Índice de Desempenho do Sistema Único de Saúde (IDSUS), as Diretrizes da Política Nacional de Saúde Bucal e uma lista de referências sobre o assunto. De 172 artigos e demais documentos pesquisados inicialmente, 35 fizeram parte da presente análise.

Este estudo integra uma pesquisa que procurou analisar o acesso à saúde bucal em um município brasileiro e foi submetido ao Comitê de Ética em Pesquisa da Faculdade de Odontologia de Piracicaba, com protocolo de aprovação número 111/2015.

\section{RESULTADOS}

Entre 2007 e 2014, foram publicados artigos sobre o tema "acesso aos serviços de saúde bucal” em todos os anos. Os anos 2008, 2009, 2011 e 2014 apresentaram maior número de publicações. Entretanto, em 2013 foi publicado apenas um artigo sobre o tema. (Quadro 1 e Tabela 1). A língua inglesa foi utilizada em três artigos.

Quadro 1: Características dos artigos de revisão da literatura sobre acesso à saúde bucal. Brasil, 2015

\begin{tabular}{|c|c|c|c|}
\hline Autor (ano)/Local & Objetivo & Tipo/Estatística & Resultado \\
\hline $\begin{array}{l}\text { Silva et al. (2007); } \\
\text { São Luís (MA) }\end{array}$ & $\begin{array}{l}\text { Analisar serviços } \\
\text { odontopediátricos }\end{array}$ & Descritivo & Atendimento restrito à atenção básica \\
\hline $\begin{array}{l}\text { Souza e Roncalli (2007); } \\
\text { Rio Grande do Norte }\end{array}$ & $\begin{array}{l}\text { Incorporação da saúde bucal } \\
\text { na ESF }\end{array}$ & Descritivo/Correlação & Problemas de territorialização \\
\hline $\begin{array}{l}\text { Bousquat et al. (2008); } \\
\text { Estado de São Paulo }\end{array}$ & $\begin{array}{l}\text { Perfil de utilização de serviços } \\
\text { de saúde }\end{array}$ & Transversal/Metodológico & Diferença: homem, escolaridade, estar empregado \\
\hline
\end{tabular}




\begin{tabular}{|c|c|c|c|}
\hline Autor (ano)/Local & Objetivo & Tipo/Estatística & Resultado \\
\hline $\begin{array}{l}\text { Freddo et al. (2008); } \\
\text { Gravataí (RS) }\end{array}$ & $\begin{array}{l}\text { Higiene bucal e utilização de } \\
\text { serviços odontológicos }\end{array}$ & $\begin{array}{c}\text { Transversal/Regressão de } \\
\text { Cox }\end{array}$ & Estilo de vida associado à utilização de serviços \\
\hline $\begin{array}{l}\text { Kramer et al. (2008); } \\
\text { Canela (RS) }\end{array}$ & $\begin{array}{l}\text { Utilização de serviços } \\
\text { odontológicos }\end{array}$ & $\begin{array}{c}\text { Transversal/Regressão } \\
\text { Logística }\end{array}$ & Fatores: sexo, mais velhos \\
\hline $\begin{array}{l}\text { Manhães e Costa (2008)*; } \\
\text { Estado do Rio de Janeiro. }\end{array}$ & $\begin{array}{l}\text { Acesso a serviços } \\
\text { odontológicos }\end{array}$ & $\begin{array}{c}\text { Transversal/Regressão } \\
\text { Logística }\end{array}$ & Jovens frequentam menos os serviços \\
\hline $\begin{array}{l}\text { Noro et al. (2008); } \\
\text { Sobral (CE) }\end{array}$ & $\begin{array}{c}\text { Utilização de serviços } \\
\text { odontológicos }\end{array}$ & $\begin{array}{c}\text { Transversal/Regressão } \\
\text { Logística }\end{array}$ & Afetada por fatores socioeconômicos \\
\hline $\begin{array}{l}\text { Rocha e Goes (2008); } \\
\text { Campina Grande (PB) }\end{array}$ & $\begin{array}{c}\text { Acesso a serviços } \\
\text { odontológicos na ESF }\end{array}$ & $\begin{array}{c}\text { Transversal/Regressão } \\
\text { Logística }\end{array}$ & Não associado a morar em área coberta pela ESF \\
\hline $\begin{array}{l}\text { Araújo et al. (2009); } \\
\text { Pelotas (RS) }\end{array}$ & Identificar padrão de utilização & $\begin{array}{c}\text { Transversal/ Regressão } \\
\text { Poisson }\end{array}$ & Escolaridade, demanda, socioeconômico \\
\hline $\begin{array}{l}\text { Baldani et al. (2009)*; } \\
\text { Estado do Paraná }\end{array}$ & $\begin{array}{l}\text { Associação entre recursos } \\
\text { financeiros e de oferta }\end{array}$ & $\begin{array}{l}\text { Ecológico/ Friedman/ } \\
\text { Spearman }\end{array}$ & $\begin{array}{l}\text { Maior provisão de recursos para municípios com } \\
\text { piores indicadores socioeconômicos }\end{array}$ \\
\hline $\begin{array}{l}\text { Camargo et al. (2009); } \\
\text { Pelotas (RS) }\end{array}$ & $\begin{array}{l}\text { Uso regular dos serviços } \\
\text { odontológicos }\end{array}$ & $\begin{array}{l}\text { Transversal/ Regressão de } \\
\text { Poisson }\end{array}$ & Uso regular pouco frequente entre os menos favorecidos \\
\hline $\begin{array}{l}\text { Fernandes et al. (2009); } \\
\text { Porto Alegre (RS) }\end{array}$ & $\begin{array}{l}\text { Padrões de utilização dos } \\
\text { serviços de saúde na ESF }\end{array}$ & $\begin{array}{l}\text { Transversal/Regressão de } \\
\text { Poisson }\end{array}$ & $\begin{array}{l}\text { Sexo feminino, mais de } 60 \text { anos, branco, menor } \\
\text { autopercepção, socioeconômico }\end{array}$ \\
\hline $\begin{array}{l}\text { Pereira et al. (2009); } \\
\text { Natal (RN) }\end{array}$ & $\begin{array}{l}\text { Impacto da ESF na utilização } \\
\text { de serviços }\end{array}$ & Ensaio Comunitário & O impacto da ESF foi positivo \\
\hline $\begin{array}{l}\text { Reis et al. (2009); } \\
\text { Grão Mogol (MG) }\end{array}$ & $\begin{array}{l}\text { Avaliar o serviço público por } \\
\text { meio da percepção do usuário }\end{array}$ & Qualitativo & $\begin{array}{l}\text { Distância, ausência de estrutura e de serviços, } \\
\text { qualidade e presença de vagas afetam o acesso }\end{array}$ \\
\hline $\begin{array}{l}\text { Baldani et al. (2010); } \\
\text { Ponta Grossa (PR) }\end{array}$ & $\begin{array}{l}\text { Fatores associados à } \\
\text { utilização de serviços }\end{array}$ & $\begin{array}{l}\text { Transversal/Regressão } \\
\text { Logística }\end{array}$ & $\begin{array}{c}\text { Cerca de } 40 \% \text { dos adultos e } 67 \% \text { dos idosos não } \\
\text { iam ao dentista há mais de 3anos }\end{array}$ \\
\hline $\begin{array}{l}\text { Souza e Chaves (2010); } \\
\text { Cidades da Bahia }\end{array}$ & $\begin{array}{l}\text { Acesso a serviços } \\
\text { especializados }\end{array}$ & $\begin{array}{l}\text { Transversal/ Exato de } \\
\text { Fisher }\end{array}$ & Baixa taxa de utilização para endodontia e cirurgia \\
\hline $\begin{array}{l}\text { Gibilini et al. (2010); } \\
\text { Estado de São Paulo }\end{array}$ & $\begin{array}{l}\text { Acesso a serviços } \\
\text { Odontológicos }\end{array}$ & Transversal/ Qui-quadrado & $\begin{array}{l}\text { Adolescentes e adultos acessaram o serviço há } \\
\text { menos de um ano, e idosos há mais de três anos }\end{array}$ \\
\hline $\begin{array}{l}\text { Celeste et al. }(2011)^{\star} \text {; } \\
\text { Brasil (regiões) }\end{array}$ & $\begin{array}{l}\text { Análise temporal de } \\
\text { procedimentos odontológicos }\end{array}$ & Ecológico/Média Móvel & $\begin{array}{l}\text { Aumento de procedimentos realizados a partir de } \\
2000 \text {. Inclusão da equipe odontológica na ESF }\end{array}$ \\
\hline $\begin{array}{l}\text { Celeste et al. }(2011)^{\star} \\
\text { Brasil e Suécia }\end{array}$ & $\begin{array}{l}\text { Uso de serviços odontológicos } \\
\text { no Brasil e na Suécia }\end{array}$ & $\begin{array}{l}\text { Transversal/Regressão de } \\
\text { Poisson }\end{array}$ & $\begin{array}{l}\text { Existem disparidades socioeconômicas na utilização } \\
\text { de serviços odontológicos nos dois países }\end{array}$ \\
\hline $\begin{array}{l}\text { Chaves et al. (2011); } \\
\text { Estado da Bahia }\end{array}$ & $\begin{array}{l}\text { Caracterizar o acesso a } \\
\text { serviços especializados }\end{array}$ & Transversal $/ \chi^{2}$ & $\begin{array}{l}\text { Houve diferença no tipo de serviço, na natureza, no } \\
\text { sexo, na escolaridade e na renda }\end{array}$ \\
\hline $\begin{array}{l}\text { Grabois et al. }(2011)^{\star} \text {; } \\
\text { Brasil/SIM/SIH }\end{array}$ & $\begin{array}{l}\text { Acesso aos serviços de saúde } \\
\text { em menores com câncer }\end{array}$ & Ecológico/Taxa & $\begin{array}{c}\text { Desigualdades no acesso para residentes em } \\
\text { regiões mais carentes }\end{array}$ \\
\hline $\begin{array}{l}\text { Haikal et al. (2011); } \\
\text { Belo Horizonte (MG) }\end{array}$ & $\begin{array}{l}\text { Autopercepção da saúde } \\
\text { bucal }\end{array}$ & Qualitativo & $\begin{array}{l}\text { A autopercepção foi preditora da procura por } \\
\text { atendimento odontológico }\end{array}$ \\
\hline $\begin{array}{l}\text { Chaves et al. (2012); } \\
\text { Municípios da Bahia }\end{array}$ & $\begin{array}{l}\text { Comparar o acesso aos } \\
\text { serviços odontológicos }\end{array}$ & Transversal $/ \chi^{2}$ & A principal barreira de acesso foi na atenção primária \\
\hline $\begin{array}{l}\text { Junqueira et al. (2012); } \\
\text { São Paulo (SP) }\end{array}$ & $\begin{array}{c}\text { Tendência de acesso aos } \\
\text { serviços }\end{array}$ & Ecológico/Regressão Linear & Idade, necessidade de exodontia, livres de cárie e local \\
\hline $\begin{array}{l}\text { Peres et al. }(2012)^{\star} ; \\
\text { Brasil. PNAD }(2003 / 2008)\end{array}$ & $\begin{array}{l}\text { Acesso aos serviços } \\
\text { odontológicos no Brasil }\end{array}$ & $\begin{array}{l}\text { Transversal/ Desvio } \\
\text { Interquartílico }\end{array}$ & Desigualdades no acesso entre os grupos sociais \\
\hline $\begin{array}{l}\text { Peres et al. }(2012)^{\star} \\
\text { Brasil VIGITEL }\end{array}$ & $\begin{array}{l}\text { Analisar falta de acesso a } \\
\text { tratamentos odontológicos }\end{array}$ & $\begin{array}{l}\text { Transversal/Regressão de } \\
\text { Poisson }\end{array}$ & $\begin{array}{l}\text { Iniquidades na utilização e no acesso para } \\
\text { mulheres, jovens, menos escolarizados e pardos }\end{array}$ \\
\hline $\begin{array}{l}\text { Viana et al. }(2012)^{\star} ; \\
\text { Pernambuco }\end{array}$ & $\begin{array}{l}\text { Identificar desigualdades } \\
\text { no acesso }\end{array}$ & $\begin{array}{l}\text { Ecológico/Intervalo } \\
\text { Interquartílico }\end{array}$ & $\begin{array}{c}\text { Baixa utilização e acesso aos serviços odontológicos } \\
\text { de forma programática na atenção básica }\end{array}$ \\
\hline $\begin{array}{l}\text { Brunhause et al. (2013); } \\
\text { Não-me-Toque (RS) }\end{array}$ & $\begin{array}{l}\text { Satisfação do usuário de } \\
\text { serviços públicos odontológicos }\end{array}$ & Transversal $/ \chi^{2}$ & 93\% satisfeitos; dificuldades gerais de acesso (vaga) \\
\hline $\begin{array}{l}\text { Fonseca et al.(2014)*; } \\
\text { Piracicaba (SP) }\end{array}$ & Oferta de serviço de urgência & $\begin{array}{l}\text { Transversal/Regressão } \\
\text { Logística }\end{array}$ & $\begin{array}{l}\text { Maior utilização do serviço de urgência por } \\
\text { residentes em áreas de maior vulnerabilidade }\end{array}$ \\
\hline $\begin{array}{l}\text { Gomes et al.(2014); } \\
\text { Maranhão (MA) }\end{array}$ & $\begin{array}{l}\text { Avaliar a utilização de serviços } \\
\text { de saúde bucal }\end{array}$ & $\begin{array}{l}\text { Transversal/Exato de Fisher } \\
\text { /Wald }\end{array}$ & $\begin{array}{c}\text { Baixa utilização dos serviços por indivíduos com } \\
\text { piores condições socioeconômicas }\end{array}$ \\
\hline $\begin{array}{l}\text { Haikal et al. (2014); } \\
\text { Montes Claros (MG) }\end{array}$ & $\begin{array}{l}\text { Acesso à informação em } \\
\text { saúde bucal }\end{array}$ & $\begin{array}{l}\text { Transversal/Regressão } \\
\text { Linear }\end{array}$ & $\begin{array}{l}\text { Adultos desinformados sobre higiene bucal } \\
\text { perderam } 2.15 \text { dentes a mais por cárie }\end{array}$ \\
\hline $\begin{array}{l}\text { Martins et al. (2014)*; } \\
\text { Brasil (SB/2003) }\end{array}$ & $\begin{array}{l}\text { Avaliação negativa de idosos } \\
\text { sobre serviços odontológicos }\end{array}$ & $\begin{array}{c}\text { Transversal/ Regressão } \\
\text { Logística }\end{array}$ & $\begin{array}{l}\text { Avaliação: serviço público e filantrópico, homens, } \\
\text { maior escolaridade, sem informação }\end{array}$ \\
\hline $\begin{array}{l}\text { Pinto et al. (2014); } \\
\text { CEO-R Sobral (CE) }\end{array}$ & $\begin{array}{l}\text { Acesso a tratamento } \\
\text { odontológico especializado }\end{array}$ & $\begin{array}{l}\text { Descritivo/ Qualitativo/Taxa } \\
\text { de Utilização }\end{array}$ & $\begin{array}{l}\text { Barreiras geográficas, financeiras e organizacionais } \\
\text { e altas taxas de faltosos }\end{array}$ \\
\hline $\begin{array}{l}\text { Rodrigues et al. (2014); } \\
\text { Montes Claros (MG) }\end{array}$ & Fatores associados & $\begin{array}{l}\text { Transversal/Regressão } \\
\text { Logística }\end{array}$ & $\begin{array}{l}\text { Idade, condição social e acesso dos pais a } \\
\text { informações preventivas }\end{array}$ \\
\hline
\end{tabular}

*Dados secundários. Estratégia Saúde da Família (ESF); Sistema de Informação sobre Mortalidade (SIM) / Sistema de Informação Hospitalar (SIH); Pesquisa Nacional por Amostra de Domicílios (PNAD); Saúde Bucal (SB); Centro de Especialidades Odontológicas Regional (CEO-R). 
A maior parte dos estudos buscou verificar a associação das desigualdades no acesso em relação a variáveis contextuais e individuais. Os estudos qualitativos abrangeram aspectos geográficos, autopercepção de saúde bucal, estrutura e qualidades dos serviços sob a perspectiva dos gestores e usuários. Em relação ao serviço público odontológico houve uma maior abordagem de aspectos de análise do acesso na atenção primária e especializada. Em 11 (31,43\%) artigos foram utilizadas fontes de dados secundários (Quadro 1).

Os estudos foram realizados nas regiões Nordeste, Sudeste e Sul, e com informações de abrangência nacional. Não foram encontrados artigos realizados nas regiões Norte e Centro-Oeste do Brasil. Houve uma diversidade na abordagem estatística com maior frequência para análises multivariadas com os testes estatísticos de Regressão Logística ( $\mathrm{n}=8 ; 22,85 \%)$ e Poisson $(\mathrm{n}=6 ; 17,14 \%)$, respectivamente (Quadro 1).

Houve uma grande diversidade no percurso metodológico (desenho) dos estudos, com uma frequência maior para estudos epidemiológicos, sendo $24(68,57 \%)$ do tipo transversal e $5(14,28 \%)$ ecológicos. Foram encontrados dois artigos $(5,72 \%)$ com abordagem qualitativa. O maior nível de evidência científica encontrado foi do tipo ensaio comunitário utilizado em apenas um $(2,85 \%)$ artigo. Não foi encontrado nenhum artigo do tipo epidemiológico longitudinal (Tabela 2).

Os principais fatores associados ao acesso à saúde e à utilização dos serviços odontológicos foram: sexo, escolaridade, empregabilidade, idade, renda, raça, autopercepção de saúde bucal, localização geográfica e acesso à informação em saúde $\mathrm{e}^{3,5-16}$.

\section{DISCUSSÃO}

O modelo teórico de acesso à saúde proposto por Andersen é empregado para estudos sobre acesso à saúde bucal em que o acesso é composto por fatores de predisposição, capacitação e necessidade em saúde ${ }^{8}$. Os modelos teóricos e a abordagem qualitativa permitem análises críticas sobre aspectos que vão desde o conceito de acesso

Tabela 1: Distribuição das publicações sobre acesso à saúde bucal entre 2007 e 2014. Brasil, 2015

\begin{tabular}{|c|c|}
\hline Período & $\mathbf{n}(\%)$ \\
\hline $2007-2008$ & $8(22,86)$ \\
\hline $2009-2010$ & $9(25,72)$ \\
\hline $2011-2012$ & $11(31,42)$ \\
\hline $2013-2014$ & $7(20,00)$ \\
\hline Total & $35(100,00)$ \\
\hline
\end{tabular}

Tabela 2: Tipos de estudo de revisão de literatura sobre acesso à saúde bucal entre 2007 e 2014. Brasil, 2015

\begin{tabular}{|c|c|}
\hline Tipo & $\mathbf{n}(\%)$ \\
\hline Transversal & $24(68,57)$ \\
\hline Ecológico & $5(14,28)$ \\
\hline Descritivo & $3(8,58)$ \\
\hline Qualitativo & $2(5,72)$ \\
\hline Ensaio comunitário & $1(2,85)$ \\
\hline Total & $35(100,00)$ \\
\hline
\end{tabular}

à saúde, formulação e execução de políticas de saúde, passando por questões da organização e assistência à saúde, até atingir a dimensão do indivíduo ${ }^{1,6,8-10}$. A reestruturação da PNSB, ocorrida a partir de 2004, pode ter desencadeado o interesse de investigação e análise; como consequência, houve o aumento no número de publicações científicas sobre o tema a partir de $2005^{2,4}$. Ademais, a diversidade metodológica e do número de variáveis apresentadas nos estudos comprometem a comparação entre os achados do estudo e, consequentemente, o estabelecimento de um padrão de estudos sobre o tema. Isso pode ser provocado pela diversidade locorregional do Brasil e pelo fato dos sistemas locais de saúde bucal estarem em diferentes estágios de descentralização ${ }^{8,11-19}$. Cabe destacar que existe uma tendência de internacionalização das revistas e pesquisas realizadas no Brasil. Os artigos publicados em língua inglesa possuem maiores chances de visibilidade, reconhecimento e citação.

\section{Conceito de acesso à saúde bucal}

O termo "acesso" é polissêmico, variou ao longo do tempo e seu conceito aponta um nível de abrangência, pluralidade, complexidade, pouca clareza e é tido como sinônimo de "porta de entrada", "utilização", "uso", "acessibilidade" ao serviço de saúde $e^{1,9-11,20}$. Em saúde bucal, existe uma tendência de ampliação do escopo do conceito de acesso como "entrada no serviço" para o "resultado" do cuidado demandado ${ }^{1}$; ou seja, um dispositivo transformador da realidade, de justiça social e de equidade ${ }^{5,20}$. Por esses motivos, não foi possível definir com clareza o conceito de acesso à saúde bucal, constituindo-se em uma limitação do estudo.

\section{Aspectos da política de saúde bucal}

Segundo Soares e Paim, o sucesso de uma política depende do acúmulo de capital teórico, instrumental e experiência das equipes dirigentes, denominado capital intelectual ${ }^{4}$. É necessário que a dimensão política tenha governabilidade, ou seja, capacidade de condução e controle do processo de governo diante de situações favoráveis ou não ao projeto ${ }^{2,4,14,21}$.

$\mathrm{O}$ elevado número de artigos encontrados pode ser explicado pelo interesse de investigar o tema e se a reestruturação da PNSB, ocorrida em 2004, impactou na melhoria do acesso à saúde bucal por meio do aumento da oferta. A macropolítica de saúde bucal, denominada PNSB - Brasil Sorridente, potencializou, para todos os níveis de atenção, o suporte nacional para o desenvolvimento de micropolíticas de saúde bucal ${ }^{2,4,14,21}$. A implementação da PNSB é expressa quando seu conteúdo propositivo ou diretriz é transformado em ação ${ }^{4}$. Essa transposição da macropolítica para a micropolítica não é harmônica e está sujeita a forças que agem no sentido de favorecer ou não a execução de ações no sentido de organizar os Sistemas Locais de Saúde Bucal (SLSB) ${ }^{2,4,21}$. Como exemplo, a fluoretação da água de abastecimento público, como estratégia macropolítica de saúde pública que, mesmo regulamentada pelo Decreto $n^{\circ}$. 76.872, de dezembro de 1975, ainda não foi implantada por muitos municípios brasileiros ${ }^{15}$. Em outras palavras, a não universalidade do acesso à água fluoretada mantém um extenso 
contingente populacional à margem do benefício ${ }^{2}$. Nesse sentido, existe a necessidade de investigar como as diretrizes da PNSB são implementadas nos SLSB.

Para Leal e Tomita ${ }^{5}$, os gestores entendem acesso como "acesso ao serviço" (grifo dos autores) e não como significado de melhores condições de saúde ${ }^{5}$. Assim, as questões referentes à saúde bucal acabam se "diluindo" no conceito geral de saúde e não sendo uma prioridade política. Isso fica visível na ausência de pautas deliberativas em saúde bucal nos Conselhos Municipais de Saúde, nas Conferências Municipais de Saúde, e na alocação de recursos financeiros, materiais e humanos para a saúde bucal ${ }^{2,4,5}$. Estudos apontam que um dos indicadores que permitem dimensionar a prioridade de determinada política é o nível de financiamento ${ }^{4,5}$. A reestruturação da PNSB trouxe avanços na questão do financiamento da saúde bucal, principalmente, a partir de incentivos financeiros em todos os níveis de atenção, como a implantação de Equipes de Saúde Bucal, Centros de Especialidade Odontológica (CEO) e Laboratórios Regionais de Prótese Dentária (LRPD) $)^{2,4,5,14}$. Entretanto, a falta de autonomia financeira sobre os recursos destinados à saúde compromete a sustentabilidade de ações de saúde bucal porque envolve a compra e a manutenção de equipamentos, materiais e insumos ${ }^{4}$.

Portanto, um dos pilares do acesso universal à saúde bucal é a integração da política de saúde bucal na agenda prioritária da política de saúde $e^{2,421}$. A literatura evidenciou a necessidade de articulação da política de saúde bucal com outras políticas e outros programas de saúde para a inclusão de grupos mais vulneráveis no acesso à saúde bucal ${ }^{4,5}$. As políticas de saúde integradas aumentam as opções para que as pessoas possam controlar melhor a sua saúde e, quando bem desenvolvidas, contribuir para que indivíduos explorem suas habilidades na concretização de projetos de vida ${ }^{2,5,14}$.

\section{Aspectos econômicos e sociais}

Para Fernandes et al., a definição de acesso à saúde bucal está relacionada com a capacidade das pessoas obterem os serviços necessários ou demandados ${ }^{22}$; ou seja, o padrão de utilização dos serviços de saúde está relacionado com as demandas/necessidades e são resultantes de fatores sociais, econômicos e culturais ${ }^{23,24}$. A capacidade de resposta a essas demandas refletem decisões equânimes, efetivas e evitam o consumo desnecessário de serviços ou a falta dos mesmos ${ }^{4,24-26}$. Isso tudo diz respeito particularmente aos municípios, pois, são o locus onde as ações de saúde são planejadas, articuladas e englobam todas as fronteiras físicas e sociais do território 5 . Assim, é patente compreender que o impacto das ações de saúde deve extrapolar a condição de saúde bucal e atingir a melhora da qualidade de vida das pessoas que habitam no território ${ }^{27-30}$. É patente reconhecer que as iniquidades no acesso à saúde bucal são resultantes de condições socioeconômicas e é necessário ampliar o acesso para setores tradicionalmente excluídos ${ }^{27,29-32}$. Para Peres et al. ${ }^{13}$, o acesso à saúde bucal significa acesso à saúde e está relacionado com a vida, a nutrição, a moradia, a renda e o acesso a bens de consumo ${ }^{25}$.

Sabe-se que o "ambiente vivido" exerce forte influência sobre a condição de saúde das pessoas ${ }^{5}$. Para compreender esse papel é preciso admitir o espaço geográfico como o local onde os sujeitos buscam suprir suas necessidades individuais, familiares e coletivas e constroem suas experiências com o mundo. A tensão na forma como os grupos sociais usam e ocupam de forma dinâmica o espaço geográfico determina, em parte, o processo saúde-doença ${ }^{4,5}$. Essa forma de analisar o espaço geográfico como um espaço socialmente determinado permite compreender que a formação das iniquidades, inclusive em saúde, são construídas a partir da ocupação desigual desse espaço geográfico ${ }^{2,4,5}$. Assim, o município acaba sendo uma expressão ou um "retrato" temporal e real da distribuição geográfica, política, econômica e social ${ }^{4,5,14}$.

Portanto, a universalização do acesso à saúde bucal depende de um cenário local ou locus ideal abrangente de componentes políticos, econômicos, sociais, de gestão da assistência, da corresponsabilização e das articulações entre os componentes e dos componentes com o meio exterior ao da saúde bucal ${ }^{4,5,14,21}$. A saúde é um valor e um estado relacionados com o desenvolvimento humano e social, que transcende a visão de oposto da doença ${ }^{4}$; o que implica na contribuição de diferentes setores, atuando sinergicamente de forma a promover impacto no cotidiano dos sujeitos ${ }^{4,5,25}$.

Chaves et al. ${ }^{6}$ estabelecem que o sucesso da reorganização das ações de saúde bucal necessita de inserção em um projeto governamental local, cuja implementação deve contar com o apoio político-popular e ser desenvolvido por profissionais capacitados ${ }^{19}$. Nesse sentido, cria-se um ambiente favorável para a mudança, com prioridade para as práticas de controle de riscos e causas, visando à autonomia dos sujeitos. Para os autores, uma gestão apoiada em ações planejadas pode dimensionar melhor os recursos disponíveis, ampliar o acesso e a cobertura e impactar favoravelmente a condição de saúde bucal ${ }^{19}$.

Assim, a condição socioeconômica de indivíduos e famílias pode determinar a capacidade de compra do serviço odontológico por plano de saúde ou de forma particular, ou seja, as desigualdades socioeconômicas limitam o acesso à saúde bucal ${ }^{4,5}$.

\section{Aspectos relacionados à gestão e assistência à saúde bucal}

Estratégias recentes em saúde bucal potencializaram a oferta de serviços decorrentes da diretriz de descentralização do SUS $4,5,13,14,17$. Para Soares e Paim, as estratégias de reorganização da PNSB evidenciaram a saúde bucal como política de saúde ${ }^{4}$. Entretanto, mesmo com a redução do percentual dos que nunca foram ao dentista no Brasil, estudos apontam que o acesso aos serviços de saúde bucal é seletivo e está relacionado com a condição econômica, a renda média e as taxas de emprego ${ }^{10,11,15,21,23,25,33}$. A necessidade de esclarecer se o aumento da oferta de serviços de saúde bucal possui reflexos no acesso ocasionou um aumento na produção científica nacional e internacional sobre o assunto, ou seja, o aumento da oferta de serviços de assistência odontológica não se traduz em melhoria do acesso ao serviço, tampouco da qualidade de vida das pessoas ${ }^{7,11,14,19,33,34}$. Apesar da ampliação da oferta de serviços assistenciais, não é acessível a todos, e a saúde bucal não se concretiza como um direito de 
todos, comprometendo o vínculo entre usuários e Equipe de Saúde Bucal (ESB) ${ }^{31,35-37}$. A literatura aponta as barreiras organizacionais, geográficas, técnicas e de recursos humanos como entraves ao acesso aos serviços odontológicos ${ }^{4,7,14,25,31}$. É necessário que a reorganização da assistência odontológica faça parte do projeto de governo local, siga as diretrizes da PNSB e melhore o acesso da população aos serviços públicos odontológicos não especializados e especializados para a garantia da integralidade do cuidado. Nesse sentido, a oferta de serviços odontológicos é uma variável que sofreu aumento na atenção básica sem que as atenções secundária e terciária acompanhassem o aumento na mesma velocidade ${ }^{10,19,23,31,33-35}$.

Estudos também apontam a existência de barreiras técnicas e geográficas como fatores limitantes do acesso à saúde, porque proporcionam um desequilíbrio na relação entre demanda e oferta e têm impacto sobre o agendamento, o tempo de espera e a formação de fila ${ }^{10,19,25}$. Soares e Paim acrescentam que a dependência de tecnologias materiais e da organização da rede de serviços de saúde bucal configuram obstáculos para o acesso à saúde bucal ${ }^{4}$.

A informação em saúde constitui outro aspecto importante para a gestão e a consolidação de um sistema de saúde público ou priva$\mathrm{do}^{5,21,38-40}$, pois é capaz de fornecer subsídios para tomada de decisão e ação gerencial, juntamente com a avaliação e o monitoramento ${ }^{21}$. Nesse contexto, os Sistemas de Informação em Saúde (SIS) são mecanismos capazes de fornecer informações epidemiológicas e assistenciais que auxiliam gestores e técnicos no direcionamento de atividades ${ }^{21,25,28}$. Dentre os diversos SIS, apenas o Sistema de Informação Ambulatorial do Sistema Único de Saúde (SIA-SUS) e o Sistema de Informação da Atenção Básica (SIAB) registram procedimentos realizados pelas equipes de saúde bucal ${ }^{21}$. Entretanto, existe uma clara divisão entre os SIS com os objetivos assistenciais e os epidemiológicos, o que compromete a apropriação das informações para a gestão e, como consequência, afeta negativamente a qualidade de serviços ofertados para a população $0^{21,25,28}$.

No Brasil, os sistemas de informação são utilizados como fonte de dados para avaliação em saúde bucal ${ }^{21,28,37}$. Entretanto, esses sistemas são frágeis no que diz respeito à qualidade da informação. Outro problema é que a avaliação em saúde bucal concentra-se na avaliação da qualidade da atenção básica e dos processos de trabalho das ESB e não considera fatores externos, que são aqueles relacionados às condições de saúde de uma determinada população $e$ a fatores demográficos, educacionais, socioculturais, econômicos, políticos, legais, tecnológicos e existenciais ou não de instituições de saúde $\mathrm{d}^{7,14,21,32,33}$.

Existem alternativas que contemplam a avaliação da assistência em outros níveis de atenção e aspectos dos serviços de saúde odontológicos $^{25,28,32,37}$, entre elas está a avaliação da cobertura por procedimentos odontológicos realizados ou potenciais ${ }^{7,14,21,32,33}$. A cobertura é um atributo básico na avaliação de um serviço de saúde, pois só se pode avaliar qualquer outro atributo se houver a oferta, a utilização de um serviço e o acesso a esse serviço $\mathrm{O}^{14,20,21,28}$. Além disso, a pesquisa sobre a satisfação dos usuários de serviços odontológicos pode detectar falhas e contribuir com informações visando ao planejamento de medidas corretivas ${ }^{32,37}$.
Portanto, é necessário compreender que os processos de avaliação expõem as diversas realidades dos serviços de saúde e de seus trabalhadores ${ }^{7,14,21,32,33,37}$. Esse fato contribui para a reflexão sobre $o$ papel que cada um possui no local onde está inserido no sistema de saúde para o aperfeiçoamento de instituições e profissionais. Apesar de considerar importantes estratégias governamentais de avaliação do sistema público e do acesso aos serviços de saúde bucal, tanto o IDSUS quanto o Programa Nacional de Melhoria do Acesso e da Qualidade da Atenção Básica (PMAQ) não foram objetos de discussão. Salientamos a necessidade de estudos futuros que utilizem indicadores de acesso potencial e obtido, presentes nos instrumentos IDSUS e PMAQ.

\section{Aspectos individuais de autopercepção, culturais e de aceitabilidade}

A “invisibilidade social” é um desafio para a organização do sistema local de saúde bucal, um entrave para a universalização do acesso equitativo e para a construção do SUS como política de inclusão social ${ }^{8,9,17,29,38-40}$. Uma das tarefas da gestão em saúde é facilitar a participação social e instrumentalizar a cultura colaborativa e do trabalho em equipe , $8,39,40^{\text {. }}$.

Cada indivíduo, inserido em um grupo social, agrega a si características e dá sentido ao que julga importante sob o ponto de vista físico, moral, intelectual e social ${ }^{17,29,38}$. Nessa direção, a centralidade do discurso atual da saúde é o autocuidado, o autogoverno e os elementos que envolvem a autonomia para a construção de sujeitos participativos ${ }^{38-39}$. Esses sujeitos são resultados de práticas sociais, culturais e racionais carregadas de significados, inclusive do conceito de saúde e de estar saudável. O indivíduo ativo é capaz de assumir responsabilidades pelas decisões tomadas ao longo da vida $^{8,9,17,29}$. Assim, a adoção de hábitos saudáveis, dietas e práticas de exercícios físicos implicam em práticas que constroem identidades e produzem indivíduos que continuamente se autovigiam ${ }^{29,38}$. Essa autovigilância pode auxiliar a detecção precoce de um quadro patológico ou identificar variáveis que interferem nas manifestações patológicas ${ }^{39,40}$, ou seja, existe um "corpo social" de fatores que pode atuar no curso da doença ou no processo saúde-doença. Além disso, há uma relação entre saúde e desigualdades nas condições de vida da população, renda, grau de desenvolvimento econômico e vínculos estabelecidos entre indivíduos e grupos.

Isso explica a autoimagem como produto do contexto social e pode ser aplicado no que os indivíduos, famílias e comunidades compreendem e ao que dão significância, como, por exemplo, o que seja saúde bucal ${ }^{29,38-40}$. Um estudo realizado com gestantes mostrou que a crença popular desaconselha ir ao dentista durante período gestacional ${ }^{38}$. A partir desse pensamento, a saúde bucal é compreendida como uma demanda social. Os próprios segmentos sociais possuem diferentes demandas por saúde que são resultantes dos processos de exclusão e que nem sempre são perceptíveis ou se tornarão políticas públicas, ${ }^{9,1729}$.

A autopercepção, portanto, é um produto social no qual a doença assume um significado específico e subjetivo para cada indivíduo, em diferentes momentos da vida, e seu impacto em saúde 
bucal está associado à qualidade de vida ${ }^{8,939,40}$. Assim, é possível afirmar que a autopercepção em saúde bucal é preditora da busca por atendimento odontológico, com impactos sobre a utilização dos serviços. Isso fica mais evidente em estudos de autopercepção de saúde bucal em idosos, em função do histórico de exposição a fatores de risco, dos motivos do uso dos serviços de saúde bucal, da autoimagem, da condição de pagar pelo serviço e do sentimento de pertencimento social ${ }^{8,9,29,39}$.

Para Abreu-de-Jesus e Assis, a aceitabilidade de um serviço de saúde abrange o subjetivo, o social e o quão significativo é a saúde para o individuo ${ }^{1}$. Em outras palavras, o uso de um serviço de saúde por si só é uma expressão positiva de acesso e está condicionado a fatores individuais ${ }^{29,38}$. Ainda segundo os autores, a informação em saúde é uma estratégia que pode transformar acesso em uso. Para Haikal et al., o acesso e a frequência da informação sobre higiene bucal estão associados à condição de perdas dentárias em adultos ${ }^{40}$. Entretanto, parte da população que não consegue assistência odontológica pública, quando procura, ou não se mobiliza na busca pelo serviço por diversos motivos, pode desistir na primeira dificuldade $e^{8,9,29,39,40}$. Esses fatos geram desconfiança na qualidade do serviço, como motivo para a busca direta por outras formas de atendimento ${ }^{8,29,38}$, isto é, as desigualdades de renda corroem o tecido social e comprometem os laços de coesão social, vínculo e confiança, traduzidos em baixos níveis de solidariedade e menor investimento nas relações sociais que comprometem indivíduos e coletividades ${ }^{8,9,17}$. Cabe também ao controle social exercer seu papel de "pressão" para produzir novas relações de organização da saúde.

\section{CONSIDERAÇÕES FINAIS}

O tema de acesso à saúde bucal tem sido objeto de discussão na literatura científica. Neste estudo foi possível fazer a análise do acesso aos serviços de saúde bucal e compreender a complexa rede de determinantes socioeconômicos, demográficos, do serviço e de pior condição de saúde bucal relacionados com o acesso à saúde bucal. Para incrementar o acesso aos serviços odontológicos, são convergentes as estratégias de redução das desigualdades socioeconômicas e ampliação de serviços odontológicos públicos que possam garantir visitas regulares ao dentista, bem como estratégias que estimulem os métodos preventivos aos agravos bucais em populações com maiores dificuldades no acesso e na utilização desses serviços.

\section{REFERÊNCIAS}

1. Abreu-de-Jesus WL, Assis MMA. Revisão sistemática sobre o conceito de acesso nos serviços de saúde: contribuições do planejamento. Ciênc Saúde Coletiva. 2010;15(1):161-70. http://dx.doi.org/10.1590/S1413-81232010000100022

2. Antunes JLF, Narvai PC. Políticas de saúde bucal no Brasil e seus impactos sobre as desigualdades em saúde. Rev Saúde Pública. 2010;44(2):360-5.

http://dx.doi.org/10.1590/S0034-89102010005000002

3. Pinheiro RS, Torres TZG. Uso de serviços odontológicos entre os estados do Brasil. Ciênc Saúde Coletiva. 2006;11(4):999-1010. http://dx.doi.org/10.1590/S1413-81232006000400021

4. Soares CLM, Paim JS. Aspectos críticos para a implementação da política de saúde bucal no município de Salvador, Bahia, Brasil. Cad Saúde Pública. 2011;27(5):966-74. http://dx.doi.org/10.1590/S0102-311X2011000500014

5. Leal RB, Tomita NE. Assistência odontológica e universalização: percepção de gestores municipais. Ciênc Saúde Coletiva. 2006;11(1):155-60.

http://dx.doi.org/10.1590/S1413-81232006000100023

6. Chaves SCL, Cruz DN, Barros SG, Figueiredo AL. Avaliação da oferta e utilização de especialidades odontológicas em serviços públicos de atenção secundária na Bahia, Brasil. Cad Saúde Pública. 2011:27(1):143-54 http://dx.doi.org/10.1590/S0102-311X2011000100015

7. Viana IB, Martelli PJL, Pimentel FC. Análise do acesso aos serviços odontológicos através do indicador de primeira consulta odontológica programática em Pernambuco: estudo comparativo entre os anos 2001 e 2009. Rev Bras Promoção Saúde. 2012;25(2): 151-60.

http://dx.doi.org/10.5020/2223

8. Andersen RM. Revisiting the Behavioral Model and Access to Medical Care: Does it Matter? J Health Social Behav. 1995;36(1):1-10. http://www.jstor.org/stable/2137284
9. Rocha RACP, Goes PSA. Comparação do acesso aos serviços de saúde bucal em áreas cobertas e não cobertas pela Estratégia Saúde da Família em Campina Grande, Paraíba, Brasil. Cad Saúde Pública. 2008;24(12):2871-80.

http://dx.doi.org/10.1590/S0102-311X2008001200016

10. Reis CJ, Martelli-Júnior H, Franco BM, Santos AA, Ramalho LMP. Avaliação do serviço de saúde bucal no município de Grão Mogol, Minas Gerais, Brasil: "a voz do usuário". Ciênc Saúde Coletiva. 2009; 14(4):1287-95

http://dx.doi.org/10.1590/S1413-81232009000400035

11. Haikal DSA, Paula AMB, Martins AMEBL, Moreira NA, Ferreira EF. Autopercepção da saúde bucal e impacto na qualidade de vida do idoso: uma abordagem quanti-qualitativa. Ciênc Saúde Coletiva. 2011;16(7):3317-29. http://dx.doi.org/10.1590/S1413-81232011000800031

12. Pinto VPT, Teixeira AH, Santos PR, Araújo MWA, Moreira MAG, Saraiva SRM. Avaliação da acessibilidade ao Centro de Especialidades Odontológicas de abrangência macrorregional de Sobral, Ceará, Brasil. Ciênc Saúde Coletiva. 2014;19(7):2235-44. http://dx.doi.org/10.1590/1413-81232014197.09862013

13. Peres KG, Peres MA, Boing AF, Bertoldi AD, Bastos JL, Barros AJD. Redução das desigualdades sociais na utilização de serviços odontológicos no Brasil entre 1998 e 2008. Rev Saúde Pública. 2012;46(2):250-8. http://dx.doi.org/10.1590/S0034-89102012000200007

14. Araújo CS, Lima RC, Peres MA, Barros AJD. Utilização de serviços odontológicos e fatores associados: um estudo de base populacional no sul do Brasil. Cad Saúde Pública. 2009;25(5):1063-72. http://dx.doi.org/10.1590/S0102-311X2009000500013

15. Chaves SCL, Soares FF, Rossi TRA, Cangussu MCT, Figueiredo ACL, Cruz DN, et al. Características do acesso e utilização de serviços odontológicos em municípios de médio porte. Ciênc Saúde Coletiva. 2012;17(11):3115-24. http://dx.doi.org/10.1590/S1413-81232012001100027 
16. Pereira CRS, Patrício AAR, Araújo FAC, Lucena EES, Lima KC Roncalli AG. Impacto da Estratégia Saúde da Família com equipe de saúde bucal sobre a utilização de serviços odontológicos. Cad Saúde Pública. 2009;25(5):985-96. http://dx.doi.org/10.1590/S0102-311X2009000500005

17. Manhães ALD, Costa AJL. Acesso a e utilização de serviços odontológicos no estado do Rio de Janeiro, Brasil, em 1998: um estudo exploratório a partir da Pesquisa Nacional por Amostra de Domicílios. Cad Saúde Pública. 2008;24(1):207-18. http://dx.doi.org/10.1590/S0102-311X2008000100021

18. Gomes AMM, Thomaz EBAF, Alves MTSSB, Silva AAM, Silva RA Fatores associados ao uso dos serviços de saúde bucal: estudo de base populacional em municípios do Maranhão, Brasil. Ciênc Saúde Coletiva.2014;19(2):629-40.

http://dx.doi.org/10.1590/1413-81232014192.23252012

19. Martins AMEBL, Jardim LA, Souza JGS, Rodrigues CAQ, Ferreira RC, Pordeus IA. A avaliação negativa dos serviços odontológicos entre idosos brasileiros está associada ao tipo de serviço utilizado? Rev Bras Epidemiol. 2014;17(1):71-90. http://dx.doi.org/10.1590/1415-790X201400010007ENG

20. Noro LRA, Roncalli AG, Mendes Júnior FIR, Lima KC. A utilização de serviços odontológicos entre crianças e fatores associados em Sobral, Ceará, Brasil. Cad Saúde Pública. 2008;24(7):1509-16. http://dx.doi.org/10.1590/S0102-311X2008000700005

21. Baldani MH, Almeida ES, Antunes JLF. Equidade e provisão de serviços públicos odontológicos no estado do Paraná. Rev Saúde Pública. 2009;43(3):446-54

http://dx.doi.org/10.1590/S0034-89102009000300008

22. Fernandes LCL, Bertoldi AD, Barros AJD. Utilização dos serviços de saúde pela população coberta pela Estratégia de Saúde da Família. Rev Saúde Pública. 2009;43(4):595-603.

http://dx.doi.org/10.1590/S0034-89102009005000040

23. Souza LF, Chaves SCL. Política Nacional de Saúde Bucal: acessibilidade e utilização de serviços odontológicos especializados em um município de médio porte na Bahia. Rev Baiana Saúde Pública. 2010;4(2):371-87.

24. Kramer PF, Ardenghi TM, Ferreira S, Fischer LA, Cardoso L, Feldens CA. Utilização de serviços odontológicos por crianças de 0 a 5 anos de idade no município de Canela, Rio Grande do Sul, Brasil. Cad Saúde Pública. 2008,24(1):150-6. http://dx.doi.org/10.1590/S0102-311X2008000100015

25. Peres MA, Iser BPM, Boing AF, Yokota RTC, Malta DC, Peres KG. Desigualdades no acesso e na utilização de serviços odontológicos no Brasil: Análise do Sistema de Vigilância de Fatores de Risco e Proteção para Doenças Crônicas por Inquérito Telefônico (VIGITEL 2009). Cad Saúde Pública. 2012;28(supl.0):S90-100. http://dx.doi.org/10.1590/S0102-311X2012001300010

26. Junqueira SR, Frias AC, Zilbovicius C, Araujo ME. Saúde bucal e uso dos serviços odontológicos em função do Índice de Necessidades em Saúde: São Paulo, 2008. Ciênc Saúde Coletiva. 2012;17(4):1015-24. http://dx.doi.org/10.1590/S1413-81232012000400023

27. Silva MCB, Silva RA, Ribeiro CCC, Cruz MCFN. Perfil da assistência odontológica pública para a infância e adolescência em São Luís (MA). Ciênc Saúde Coletiva. 2007;12(5):1237-46. http://dx.doi.org/10.1590/S1413-81232007000500021
28. Celeste RK, Vital JF, Junger WL, Reichenheim ME. Séries de procedimentos odontológicos realizadas nos serviços públicos brasileiros, 1994-2007. Ciênc Saúde Coletiva. 2011;16(11):4523-32. http://dx.doi.org/10.1590/S1413-81232011001200025

29. Freddo SL, Aerts DRGC, Abegg C, Davoglio R, Vieira PC, Monteiro L. Hábitos de higiene bucal e utilização de serviços odontológicos em uma cidade da região sul do Brasil. Cad Saúde Pública. 2008;24(9):1991-2000. http://dx.doi.org/10.1590/S0102-311X2008000900005

30. Celeste RK, Nadanovsky P, Fritzell J. Trends in socioeconomic disparities in the utilization of dental care in Brazil and Sweden. Scand J Public Health. 2011;39(6):640-8.

http://dx.doi.org/10.1177/1403494811414246

31. Grabois MF, Oliveira EXGO, Carvalho MS. Childhood cancer and pediatric oncologic care in Brazil: access and equity. Cad Saúde Pública. 2011;27(9):1711-20.

http://dx.doi.org/10.1590/S0102-311X2011000900005

32. Bousquat A, Alves MCGP, Elias PE. Utilização do Programa de Saúde da Família em regiões metropolitanas: abordagem metodológica. Rev Saúde Pública. 2008;42(5):903-6. http://dx.doi.org/10.1590/S0034-89102008005000044

33. Souza TMS, Roncalli AG. Saúde Bucal no Programa Saúde da Família: uma avaliação do modelo assistencial. Cad Saúde Pública. 2007;23(11):2727-39.

http://dx.doi.org/10.1590/S0102-311X2007001100020

34. Fonseca DAV, Mialhe FL, Ambrosano GMB, Pereira AC, Meneghim MC. Influência da organização da atenção básica e das características sociodemográficas da população na demanda pelo pronto atendimento odontológico municipal. Ciênc Saúde Coletiva. 2014;19(1):269-78. http://dx.doi.org/10.1590/1413-81232014191.2048

35. Camargo MBJ, Dumith SC, Barros AJD. Uso regular de serviços odontológicos entre adultos: padrões de utilização e tipos de serviço. Cad Saúde Pública. 2009;25(9):1894-1906. http://dx.doi.org/10.1590/S0102-311X2009000900004

36. Rodrigues LAM, Martins AMEBL, Silveira MF, Ferreira RC, Souza JGS, Silva JM, et al. Uso de serviços odontológicos entre préescolares: estudo de base populacional. Ciênc Saúde Coletiva. 2014;19(10):4247-56.

http://dx.doi.org/10.1590/1413-812320141910.13382013

37. Brunhauser AL, Magro ML, Neves M. Avaliação de serviços de saúde bucal: um estudo comparativo. RFO. 2013;18(1):24-31. http://dx.doi.org/10.5335/rfo.v18i1.2850

38. Baldani MH, Brito WH, Lawder JAC, Mendes YBE, Silva FFM, Antunes JLF. Determinantes individuais da utilização de serviços odontológicos por adultos e idosos de baixa renda. Rev Bras Epidemiol. 2010;13(1):150-62. http://dx.doi.org/10.1590/S1415-790X2010000100014

39. Gibilini C, Esmeriz CEC, Volpato LF, Meneghim ZMAP, Silva DD, Sousa MLR. Acesso a serviços odontológicos e auto-percepção da saúde bucal em adolescentes, adultos e idosos. Arq Odontol. 2010;46(4):213-23.

40. Haikal DSA, Martins AMEBL, Aguiar PHS, Silveira MF, Paula AMB, Ferreira EF. O acesso à informação sobre higiene bucal e as perdas dentárias por cárie entre adultos. Ciênc Saúde Coletiva. 2014;19(1):287-300. http://dx.doi.org/10.1590/1413-81232014191.2087 